\title{
Vision Loss from Atypical Optic Neuritis: Patient and Physician Perspectives
}

Tasanee Braithwaite $\cdot$ Nils Wiegerinck · Axel Petzold ·

Alastair Denniston

Received: January 13, 2020 / Published online: March 21, 2020

(C) The Author(s) 2020

\section{ABSTRACT}

This article, co-authored by a patient affected by bilateral, recurrent, atypical optic neuritis, and clinicians, discusses the mental burden of living

Enhanced Digital Features To view digital features for this article go to https://doi.org/10.6084/m9.figshare. 11941020.

T. Braithwaite $(\bowtie) \cdot$ A. Petzold

Neuro Ophthalmology Department, Moorfields Eye Hospital, London, UK

e-mail: tasaneebraithwaite@gmail.com

T. Braithwaite $\cdot$ A. Denniston

Ophthalmology Department, University Hospitals

Birmingham, Birmingham, UK

T. Braithwaite $\cdot$ A. Petzold

Neuro Ophthalmology Department, National

Hospital for Neurology \& Neurosurgery, London, UK

N. Wiegerinck

Amsterdam, The Netherlands

A. Petzold

Institute of Neurology, University College London, London, UK

A. Petzold · A. Denniston

National Institute for Health Research (NIHR)

Biomedical Research Centre Based at Moorfields Eye Hospital NHS Foundation Trust, UCL Institute of Ophthalmology, London, UK

\section{A. Denniston}

Institute of Inflammation and Ageing, University of Birmingham, Birmingham, UK with uncertainty and the possibility of further sight loss, along with the side effects of treatment. The patient shares some of the challenges, coping strategies, and the value they found in creating and participating in a patient support group. The physicians consider whether current clinical measures adequately capture the outcomes that matter to patients and discuss the role for patient-reported outcome measures (PROMs). We identify technological advances that are lowering traditional barriers to the use of PROMs in research and routine clinical care and look towards new PROM instruments enhancing shared patient-physician care in the future.

\section{PLAIN LANGUAGE SUMMARY}

In this patient-physician perspective article, we share the story of a patient affected by an autoimmune disease that attacks the nerves connecting the eyes and the brain and reflect back physicians' perspectives on the disease and the patient's experience of it. In a compelling account, we gain some understanding of what it might be like to live with the fear of unpredictable episodes of sudden, recurrent sight loss and the important impacts that this has on a patient's life and mental wellbeing. We recognize that the outcome metrics that physicians 
usually focus on, such as measurement of vision and imaging of the optic nerve, do not fully capture the outcomes that most matter to the patient. We explore patient-reported outcome measures that go some way towards bridging this gap. Finally, we consider the technological advances that will make more comprehensive capture of the patient experience a reality in future clinical practice and research, supporting both patients and physicians to optimize shared care.

Keywords: Chronic relapsing inflammatory optic neuropathy (CRION); Optic neuritis; Patient-reported outcome measure (PROM); Quality of life

\section{Key Summary Points}

Patient perspectives on the impact of optic neuritis have been seldom reported in the medical literature.

There is growing recognition of the importance of the 'patient voice' in ophthalmology research and clinical practice.

Patient accounts provide valuable insights into the outcomes and metrics that matter most to patients.

A few patient-reported outcome measures have been developed in neuroophthalmology, and in multiple sclerosis and neuromyelitis optica spectrum disorder specifically. In our view, these are not yet psychometrically optimized for use in optic neuritis patients in routine clinical practice or clinical trials.

Technological developments are overcoming traditional barriers to the routine use of comprehensive patientreported outcome measures.

\section{PATIENT PERSPECTIVE}

I was 28 when, 12 years ago, I felt a twinge in my right eye after a mild flu. Not thinking anything of it, I gave it little thought until, after 2 weeks, the pain had become constant. After ineffective antibiotic treatment, I was referred to the hospital, where they established I had optic nerve inflammation and only $40 \%$ of the vision in my right eye remained. With intravenous prednisone therapy my eyesight improved up to $80 \%$, but after 2 weeks it dropped again, this time to no perception of light in just $36 \mathrm{~h}$. Subsequent intravenous prednisone did not help. An MRI (magnetic resonance imaging scan) ruled out multiple sclerosis and I was told this was an episode of bad luck: My immune system had probably confused my eye nerve with a virus and attacked it. Living with only one seeing eye is actually not a big change (unless you have an aspiring squash career, which I did not), and I got used to it in a matter of weeks. But 7 years after this episode, I felt the same tingling sensation in my left eye. Losing one eye is fine, but going blind in both is a completely different matter. I was diagnosed with a chronic relapsing inflammatory optic neuropathy (CRION). Intravenous prednisone when attacks flare up and daily prednisone tablets have since helped to retain the eyesight in my left eye. In spite of this treatment, I get two or three attacks per year, and although repeat OCT (optical coherence tomography) scans show that the nerve damage hasn't deteriorated much, so far I've been lucky.

The above is roughly what I tell people when they ask me about it. The current medical world is mostly focused on traditional measurable metrics. For me, the key metrics my doctors use include visual acuity, OCT scans, and intraocular pressure. But there are other unquantifiable factors, such as mental aspects, convenience of treatment, and quality of life, that in the current medical climate get less attention. In my experience this can leave important patient questions unaddressed, in turn missing an opportunity to do better in helping patients deal with their disease and its treatment. 
Take mental stress for instance. In my case, losing sight in one eye was not a big deal. But losing sight in my other eye would drastically change my life. I have a 16-month-old son at home, and I want to see him growing up. So naturally, I want to do all I can to keep my eyesight, and my independence. I'm convinced human beings in general aren't good at dealing with the unknown. We have a natural tendency to look for (a sense of) control. We want to eliminate the distress of the unknown, so we're always looking for cognitive closure. And when the medical world cannot help you in achieving this sense of control, you start to venture out by yourself to see what you can do. The overarching question for me is, "what is causing this, and what can I do to limit the attacks and keep my eyesight?" Given the rising incidence of autoimmune diseases in the population, it seems to point at environmental factors (vs. genetic factors), so I wonder what I can do to change my environment, to balance my immune system again. I've looked in various directions: Are autoimmune disorders related to stress? If so, how can I minimize stress? What role does diet play? What is the relationship between gut health and the immune system? Can more physical activity and more sleep have a positive effect?

To find answers to these questions I started to read up online. But as most doctors have probably experienced with patients, Google will find any correlation you want. Confirmation bias thrives online. And it's very easy to get down a rabbit hole or into an echo chamber on topics that might lack a scientific evidence base. I started changing my routines. What I eat, when I eat, when and how much I work out, and my sleeping patterns. With each change I felt hopeful about finding the holy grail, but then the next attack would come, prompting me to re-evaluate and correct course.

For most patients I'm convinced the mental burden is the most difficult part of their disease. Regardless if it's autoimmune disease, cancer, or even a complicated fracture. Variations on "What will happen to me in the future?" For me the constant question is, "Will I go blind", and the underlying question is, "How is my eyesight right now? Am I seeing less or more?" When I have an attack, I'm re-evaluating my eyesight up to 1000 times a day: "Should I be able to read that sign across the road? Is it the lighting in the room or is it my vision?" And so on. This "Am I going blind" Sword of Damocles completely occupies me. I'm afraid my body will not respond quickly enough to treatment of an attack, which will further damage my optic nerve. But at the same time, I do not want to increase the dose of my medication unnecessarily, as this could cause more side effects. When I go to clinic for a check-up, the visual acuity and OCT results do not always match up with my experience of the quality of my eyesight. All the while, I try not to let it occupy me too much, as it puts more mental stress on my body, and it feels like this might further create a negative spiral. But it's like the pink elephant in the room: impossible not to think about it when someone tells you not to! I'm confronted with the quality of my eyesight every waking moment, so it's more difficult to shut down that thought process. These mental struggles are inherent to all diseases-my situation is far from unique. And I understand that some doctors might think it's futile to discuss this aspect with the patient as there are often no clear answers or solutions to give. My point is that patients will venture out themselves if not discussed. Our natural tendency is to want to fill the void of not knowing, and putting this mental aspect more central in the treatment/communication with the patient can help patients, even if the answers are not there.

I feel lucky to have a great relationship with my neuro-ophthalmologist, and this has helped me a lot. Through discussing the mental aspects, my doctor floated the idea of creating a patient group to create a space where similar patients can find information and share experiences. Experiences of what has helped them personally, and what hasn't. We share thought processes around dealing with the disease and keep each other informed of developments in treatments. The patient group helps to sift through the forest of articles, theories, and possible solutions and helps us to better avoid the confirmation bias when venturing out to find answers alone. It has helped us share best practices. One example, for instance, is how to 
lose water retained by your body due to the prednisone. I experimented with a cleanse diet of 1 week where I only ate greens, nuts, and apples. No caffeine, carbs, alcohol, salt, sugar, and fats. I lost nearly a kilo a day throughout the cleanse, and repeat it every so often. It helps me to lower my internal pressure and avoids me looking bloated. Though small, these are important victories in dealing with this disease and having a patient group is a great way to be able to share and discuss.

\section{PHYSICIAN PERSPECTIVE}

"... and now come and sit beside me for a little while, and touch me with your hand.

For I cannot see you, Charley; I am blind."

These are the words of a young woman to her child, after discovering that she had lost her vision. The quote is taken from the novel Bleak House by Charles Dickens (1812-1870) [1]. In this novel, the time course and clinical signs of a disease process, which could have resulted from simultaneous bilateral optic neuritis or chiasmitis, are carefully married up chronologically with the emotions and social interactions of the main character, Esther Summerville. This story predates discovery of the ophthalmoscope, the clinical definition of optic neuritis, and any form of treatment. Yet the human thoughts and emotions are little different today: "I have a 16-month-old child"; "I want to see him grow up"; "Will I go blind?" The last question is difficult to answer, for whilst we now have treatment options, they may fail in some individuals and in differing types of optic neuritis.

Optic neuritis is an important cause of potentially irreversible vision impairment [2]. The most typical cause is a demyelinating inflammatory lesion, which can be isolated or associated with multiple sclerosis. Patients with typical optic neuritis usually experience good recovery of their visual acuity in the first month after the vision loss, without any treatment, although the quality of the recovered vision, and especially the contrast sensitivity or colour perception, may be worse than before. In contrast, 'atypical' causes of optic neuritis may present with more severe vision loss (e.g. visual acuity worse than $6 / 60$ at onset), bilateral involvement, and/or no pain on eye movement. These cases usually require urgent high-dose corticosteroid therapy, and some, including CRION, are 'steroid dependent', meaning that the disease often relapses when the steroid dose is weaned or discontinued. Therefore, secondline steroid-sparing agents (e.g. azathioprine) are required in these patients, aiming to reduce the risk of steroid side effects and further vision loss or neurological morbidity resulting from relapses [3]. Plasma exchange, intravenous immunoglobulin, and biological therapies are also used.

The patient outlines how the impacts of optic neuritis and its treatment extend far beyond the limited visual function measures and OCT imaging parameters monitored in clinic to encroach upon many different domains of quality of life. There is growing recognition of the value of, and need for, patient-reported outcome measures in medicine and ophthalmology $[4,5]$. Few tools have been developed to date for neuro-ophthalmology, and optic neuritis specifically. For example, the neuro-ophthalmic supplement to the National Eye Institute Visual Functioning Questionnaire (NEI-VFQ) includes ten questions [6]. These include questions on visual symptoms, performance in vision-related daily activities, and appearance. This tool has been used in optic neuritis clinical trials [7]. However, it covers only three domains of quality of life, and the composite summary score lacks psychometric validity, and is not amenable to parametric statistical analysis [8, 9]. More recently, a 46-item instrument was developed for neuromyelitis optica spectrum disorder, one rare cause of optic neuritis [10]. This includes questions on a large number of quality of life domains, including vision and vision-related functioning, impacts on life goals and roles, general health-related functioning, mobility, bladder function, bowel function, sexual function, mood, pain, general fatigue, and cognition. A systematic review of PROM instruments applicable in optic neuritis is underway and will 
report on the psychometric properties and quality of available instruments [11].

To overcome some of the psychometric limitations of PROMs assessing quality of life in other ophthalmic diseases (e.g. diabetic retinopathy and hereditary retinal disease), comprehensive, Rasch-validated multi-dimensional instruments with interval scoring are being developed $[12,13]$. These capture over ten quality of life domains that matter to patients, have interval scale properties, and yield parametrically distributed continuous outcome measures. These 'third-generation' PROM tools have the potential to transform clinical trial outcome measures and routine patient care [5].

Technological advances are rapidly addressing the traditional barriers to the integration of PROMs in clinical medicine. PROMs can now be administered on phones, tablets, and computers, with software to overcome low vision, literacy, and language barriers to completion, with computer-adaptive testing to reduce time response burden, and utilizing cloud-based data storage and integration platforms to link PROM outcomes into the electronic medical record for virtual review by physicians [14]. Iterative, patient-involved development of such PROM tools has the potential to transform both patient and physician experience, enhancing the potential for truly shared care. This article is based on previously conducted studies and does not contain any studies with human participants or animals performed by any of the authors.

\section{ACKNOWLEDGEMENTS}

We thank the patient who participated in and co-authored this study.

Funding. No funding or sponsorship was received for this study or publication of this article.

Authorship. All named authors meet the International Committee of Medical Journal Editors (ICMJE) criteria for authorship for this article, take responsibility for the integrity of the work as a whole, and have given their approval for this version to be published.

Authorship Contributions. Substantial contributions to the conception or design of the work: Alastair Denniston, Tasanee Braithwaite. Acquisition, analysis or interpretation of data: Nils Wiegerinck, Tasanee Braithwaite, and Axel Petzold. Drafting the work or revising it critically for important intellectual content, the final approval of the version to be published, and the agreement to be accountable to all aspects of the work was completed by Tasanee Braithwaite, Nils Wiegerinck, Axel Petzold, and Alastair Denniston.

Disclosures. Tasanee Braithwaite, Nils Wiegerinck, Axel Petzold, and Alastair Denniston have no conflicts of interest to declare. Axel Petzold and Alastair Denniston were supported by the National Institute for Health Research (NIHR) Biomedical Research Centre based at Moorfields Eye Hospital NHS Foundation Trust and UCL Institute of Ophthalmology. The views expressed are those of the authors and not necessarily those of the NHS, the NIHR, or the Department of Health. Tasanee Braithwaite was supported by the patient charity Olivia's Vision.

Compliance with Ethics Guidelines. This article is based on previously conducted studies and does not contain any studies with human participants or animals performed by any of the authors.

Peer Review. Please note, contrary to the journal's standard single-blind peer review process, as a commentary this article underwent review by a member of the journal's Editorial Board.

Data Availability. Data sharing is not applicable to this article as no datasets were generated or analysed during the current study.

Open Access. This article is licensed under a Creative Commons Attribution-NonCommercial 4.0 International License, which permits any non-commercial use, sharing, adaptation, distribution and reproduction in any medium 
or format, as long as you give appropriate credit to the original author(s) and the source, provide a link to the Creative Commons licence, and indicate if changes were made. The images or other third party material in this article are included in the article's Creative Commons licence, unless indicated otherwise in a credit line to the material. If material is not included in the article's Creative Commons licence and your intended use is not permitted by statutory regulation or exceeds the permitted use, you will need to obtain permission directly from the copyright holder. To view a copy of this licence, visit http://creativecommons.org/licenses/by$\mathrm{nc} / 4.0 /$.

\section{REFERENCES}

1. Petzold A. Optic neuritis: another dickensian diagnosis. Neuroophthalmology. 2013;37(6):247-50.

2. Petzold A, Braithwaite T, van Oosten BW, et al. Case for a new corticosteroid treatment trial in optic neuritis: review of updated evidence. J Neurol Neurosurg Psychiatry. 2020;91(1):9-14.

3. Jurynczyk M, Messina S, Woodhall MR, et al. Clinical presentation and prognosis in MOG-antibody disease: a UK study. Brain. 2017;140(12):3128-38.

4. Dean S, Mathers JM, Calvert M, et al. "The patient is speaking": discovering the patient voice in ophthalmology. Br J Ophthalmol. 2017;101(6):700-8.

5. Braithwaite T, Calvert M, Gray A, Pesudovs K, Denniston AK. The use of patient-reported outcome research in modern ophthalmology: impact on clinical trials and routine clinical practice. Patient Relat Outcome Meas. 2019;10:9-24.

6. Raphael BA, Galetta KM, Jacobs DA, et al. Validation and test characteristics of a 10-item neuro- ophthalmic supplement to the NEI-VFQ-25. Am J Ophthalmol. 2006;142(6):1026-35.

7. Petrillo J, Balcer L, Galetta S, Chai Y, Xu L, Cadavid D. Initial impairment and recovery of vision-related functioning in participants with acute optic neuritis from the RENEW trial of opicinumab. J NeuroOphthalmol. 2019;39(2):153-60.

8. Khadka J, McAlinden C, Gothwal VK, Lamoureux EL, Pesudovs K. The importance of rating scale design in the measurement of patient-reported outcomes using questionnaires or item banks. Invest Ophthalmol Vis Sci. 2012;53(7):4042-54.

9. Kuspinar A, Mayo NE. A review of the psychometric properties of generic utility measures in multiple sclerosis. PharmacoEconomics. 2014;32(8):759-73.

10. Moore P, Jackson C, Mutch K, et al. Patient-reported outcome measure for neuromyelitis optica: pretesting of preliminary instrument and protocol for further development in accordance with international guidelines. BMJ Open. 2016;6(9):e011142.

11. Braithwaite TLX, Panthagani J, Aiyegbusi OL, Bayliss S, Calvert M, Pesudovs K, Moore D, Denniston A (2019) Measurement properties of patient-reported outcome measures (PROMs) used in adult patients with ocular immune-mediated inflammatory disease (uveitis, scleritis or optic neuritis): a systematic review. 2019. https://www.crd.york.ac.uk/ PROSPERO/display_record.php?RecordID $=151652$. Accessed 11 Feb 2020.

12. Khadka J, Fenwick E, Lamoureux E, Pesudovs K. Methods to develop the eye-tem bank to measure ophthalmic quality of life. Optometry Vis Sci. 2016;93(12):1485-94.

13. Pesudovs K, Khadka J, Senthil MP, et al. Diseasespecificity and internationalisation of ophthalmic quality of life item banks. Investigative Ophthalmology and Visual Science Conference 2018; 59(9).

14. Aiyegbusi OL. Key methodological considerations for usability testing of electronic patient-reported outcome (ePRO) systems. Qual Life Res. 2020;29(2): 325-33. 